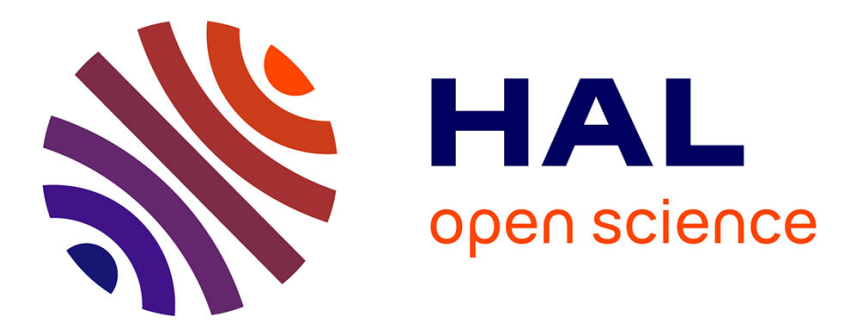

\title{
Aux marges de la ville, les camps de réfugiés palestiniens à Tyr \\ Kamel Doraï
}

\section{To cite this version:}

Kamel Doraï. Aux marges de la ville, les camps de réfugiés palestiniens à Tyr. Outre-terre. Revue européenne de géopolitique, 2006, 13, pp.373-389. 10.3917/oute.013.0373 . halshs-00416199

\section{HAL Id: halshs-00416199 \\ https://shs.hal.science/halshs-00416199}

Submitted on 13 Sep 2009

HAL is a multi-disciplinary open access archive for the deposit and dissemination of scientific research documents, whether they are published or not. The documents may come from teaching and research institutions in France or abroad, or from public or private research centers.
L'archive ouverte pluridisciplinaire HAL, est destinée au dépôt et à la diffusion de documents scientifiques de niveau recherche, publiés ou non, émanant des établissements d'enseignement et de recherche français ou étrangers, des laboratoires publics ou privés. 


\begin{abstract}
"Espaces de transit et d'attente, [les camps] s'organisent comme des "villes" sans pour autant être dotés d'un projet urbain dans la mesure où tout est conçu pour ne pas durer. Ils se stabilisent et durent."
\end{abstract}

\title{
1. Introduction.
}

La société palestinienne, dispersée depuis 1948, s'est concentrée dans des camps de réfugiés au Moyen-Orient qui sont devenus le symbole même de cette diaspora. La dynamique territoriale des diasporas ainsi que leur perception particulière de l'espace, en font des organisations socio-spatiales singulières, unies autour d'un territoire mythique, et dispersée sur différents espaces nationaux. L'identité palestinienne qui s'exprime aujourd'hui dans l'exil, et dont les camps sont l'une des incarnations spatiales les plus visibles et les plus représentatives, repose sur deux dynamiques différentes qui se croisent pour dessiner les contours de la communauté palestinienne : une frontière interne, l'histoire palestinienne, et une frontière externe, son rapport avec les sociétés d'accueil. ${ }^{3}$ Les camps de réfugiés, à l'origine conçus comme des structures temporaires ${ }^{4}$, se sont inscrits dans le paysage urbain libanais de façon durable, pour faire faire partie intégrante des principales agglomérations littorales. La place des camps de réfugiés dans la ville demeure cependant singulière, surtout au sud Liban, où ces derniers sont soumis à un strict contrôle de l'armée libanaise et où une certaine extra-territorialité demeure.

Les réfugiés palestiniens ont développé dans l'exil un rapport particulier à l'espace qui s'ancre dans les camps de réfugiés. Ces espaces, symboles de la diaspora, sont l'expression même de la géographie palestinienne de l'exil parce qu'ils rappellent le statut de réfugié des Palestiniens, qu'ils sont des lieux d'expression et de recomposition de leur identité et qu'ils traduisent les contraintes exercées par leurs pays d'accueil. La singularité des pratiques spatiales des réfugiés s'exprime donc par leur mode d'implantation particulier dans leurs espaces d'accueil.

\footnotetext{
${ }^{1}$ Chargé de recherche à Migrinter (CNRS - UMR 6588, Poitiers) Mohamed-Kamel.Dorai@univ-poitiers.fr

${ }^{2}$ M. Agier, "Le son de la guerre. Expériences africaines de l'errance, des frontières et des camps", in Politix, vol. 24, n69, 2004, pp.83.99.

${ }^{3}$ Nous reprenons ici la distinction opérée par Danielle Juteau (L'ethnicité et ses frontières, Montréal, Les Presses de l'Université de Montréal, 1999).

${ }^{4}$ L'UNRWA estime qu'en 1959 l'ensemble des tentes ont disparu des camps et que l'habitat en dur s'y est substitué.
} 
La diaspora palestinienne compte aujourd'hui près de quatre millions de réfugiés. Plus de $90 \%$ résident dans les pays frontaliers d'Israël et des Territoires palestiniens (Carte 1). Cette relative concentration spatiale ne doit pas masquer les importantes différences qui caractérisent chacune des communautés palestiniennes dispersées. En ce sens les Palestiniens du Liban présentent toute une série de particularités liées à l'organisation socio-spatiale singulière de leur pays d'accueil. De petite taille, le Liban est fait de contrastes : une géographie accidentée où les espaces montagneux qui constituent l'essentiel du territoire dominent une étroite plaine littorale; une société urbaine, littorale et commerçante qui coexiste avec un monde rural, certes de plus en plus marginalisé, mais qui marque toujours de son empreinte le Liban actuel; et un pays longtemps parmi les plus prospères et dynamiques de la région qui porte aujourd'hui les stigmates d'une longue guerre civile qui a détruit l'essentiel de son potentiel économique.

Les camps, longtemps laboratoires de développement de l'identité et des institutions palestiniennes, se trouvent aujourd'hui être des lieux de repli et d'attente. L'activité économique productive tend à disparaître de ces espaces et seuls les petits commerces s'y développent. Dans la région de Tyr où réside une part importante de la population palestinienne du Liban, on assiste à un phénomène paradoxal. Certains camps et groupements ${ }^{5}$ proches de la ville de Tyr tendent à "s'intégrer" de plus en plus au tissu urbain alors que dans le même temps la législation et le contrôle qui leur sont imposés les stigmatisent et les isolent de leur environnement direct. Ces espaces apparaissent de plus en plus comme des zones marginalisées dans un environnement urbain en mutation rapide.

\footnotetext{
${ }^{5}$ La distinction entre camp et groupement repose sur le statut juridique de ces espaces. Alors que le camp est un espace reconnu par l'Etat d'accueil dont la gestion est confiée à l'UNRWA, le groupement (tajamouaa en arabe) est une zone d'habitation informelle créée par les réfugiés eux-mêmes qui ne relève pas du mandat de l'UNRWA.
} 


\section{Carte 1}

Les réfugiés palestiniens immatriculés à l'UNRWA en 2003

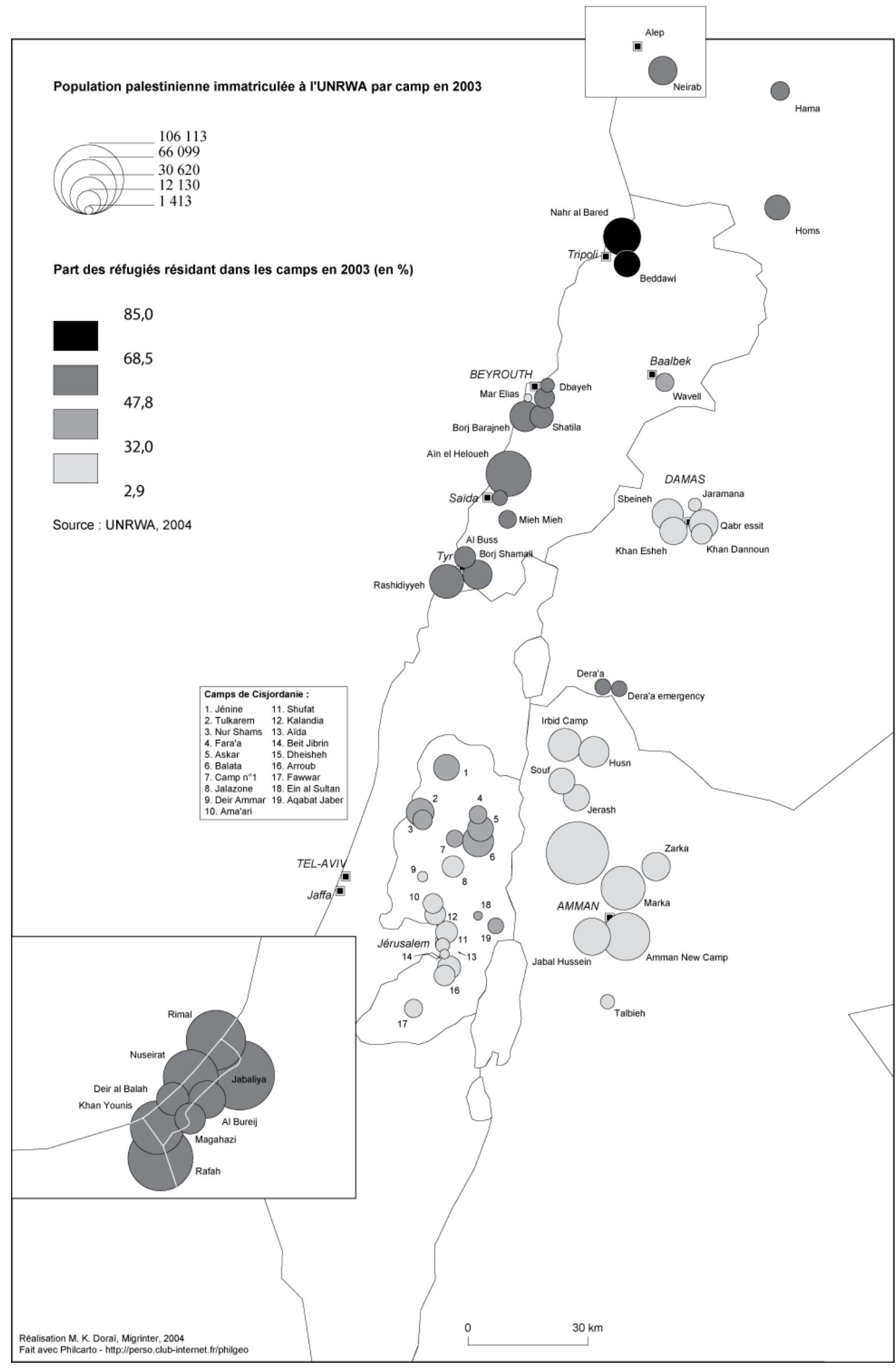




\section{Les Palestiniens du Liban, une population marginalisée.}

\subsection{Un statut précaire.}

Avec près de 400000 individus $^{6}$, il s'agit d'une des principales communautés qui réside en dehors des frontières de la Palestine du mandat britannique, derrière la Jordanie et à égalité avec la Syrie. La communauté palestinienne qui réside au Liban est aujourd'hui l'une de celles dont l'avenir est le plus problématique ${ }^{7}$.Dans les années soixante-dix, les camps et les groupements palestiniens ont connu un important développement spatial, surtout dans le sud où les institutions diasporiques étaient très présentes. On assiste alors à une densification et à une amélioration qualitative de l'habitat, à la mise en place d'infrastructures de base et à une diversification des activités économiques qui permettent une progression substantielle du niveau de vie des réfugiés. Ces derniers sont confrontés aujourd'hui à une double contrainte : un processus de paix en crise à l'échelle régionale, qui a longtemps occulté le volet des réfugiés de 1948, et une marginalisation socio-politique de plus en plus prononcée dans leur Etat d'accueil. La présence palestinienne au Liban est faite de contradictions et de ruptures. Les Accords de Taëf (1989) qui mettent fin au conflit libanais confirment le fonctionnement communautaire de l'Etat libanais qui ne laisse aucune place à la population palestinienne. Dans ce pays, les Palestiniens sont relégués aux marges de la société. Depuis le milieu des années quatre-vingt, les gouvernements libanais successifs ont réactivé toute une série de lois qui restreignent fortement l'accès des Palestiniens au marché du travail ${ }^{8}$, aux services sociaux, à l'éducation et à la liberté de circulation vers l'étranger. Cette politique se concrétise par un contrôle très strict des camps de réfugiés, surtout dans le sud, par l'impossibilité de se voir délivrer un permis de travail et par une politique de visa d'entrée et de sortie sur le territoire libanais très restrictive. Les camps et les groupements palestiniens, partiellement détruits pendant la guerre civile, ne sont pas intégrés dans les plans de reconstruction élaborés par l'Etat libanais. Faute de pouvoir rénover leur logement parce qu'ils n'en n'ont ni les

\footnotetext{
${ }^{6}$ Il existe au Liban un débat sur le nombre de Palestiniens présents sur le sol libanais, les chiffres variant de 400000 à 250 000. Cette différence s'explique en partie par le mode de calcul adopté, qui reprend soit le nombre de réfugiés inscrits à l'UNRWA (soit 399152 au 31 décembre 2004), soit le nombre estimé de personnes réellement présentes sur le sol libanais aujourd'hui, qui étant donné l'importante émigration se situerait autour de 250000 individus, sans que cette émigration puisse être quantifiée de manière précise. Les chiffres de l'UNRWA accessibles et comparables dans le temps et qui serviront le plus probablement de références dans les négociations sur les réfugiés, sont utilisés ici.

${ }^{7}$ S. Dupuis, "Palestiniens au Liban : une précarité source d'instabilité" in Les cahiers de l'Orient, nº35, troisième trimestre, 1994, p. 121-131; S. Haddad, The Palestinian Impasse in Lebanon: The Politics of Refugee Integration, Sussex Academic Press, 2003; N. Salam, "Quel avenir pour les Palestiniens du Liban ?" in Revue d'études palestiniennes, $\mathrm{n}^{\circ}(53)$ 1, nouvelle série, 1994, p. 9-20.

${ }^{8}$ Plus de cinquante professions sont interdites aux Palestiniens. On peut citer par exemple celles d'avocat, médecin, pharmacien, ingénieur (S. Al-Natour, "The legal status of Palestinians in Lebanon" in Journal of Refugee Studies, vol. 10, n³, Septembre 1997, pp. 368-371). Cette interdiction a été partiellement levée par le ministre du travail libanais Trad Hamadé en juin 2005, mais ne concerne pas les emplois de cadre les professions libérales ou celles citées précédemment (Al Nahar, 28 juin 2005 et L'Orient - Le Jour, 28 juin 2005).
} 
moyens ni le droit, l'habitat est en voie de précarisation, surtout dans les groupements où l'UNRWA ${ }^{9}$ est absente. Aujourd'hui les Palestiniens du Liban se trouvent donc privés de toute perspective d'avenir, alors même que leur précarité s'accroît : en effet, près des deux tiers des réfugiés vivent actuellement en dessous du seuil de pauvreté. ${ }^{10}$

La question des réfugiés palestiniens concerne le pouvoir libanais parce qu'elle touche à la structure démographique de sa population. Au Liban les institutions actuelles sont fondées sur une balance communautaire héritée du recensement de population de 1932, ainsi que des rapports de pouvoir de l'époque. La parité entre chrétiens et musulmans est aujourd'hui obsolète. La présence de près de 400000 réfugiés palestiniens, dont l'écrasante majorité est de confession musulmane sunnite, représente un défi pour les autorités de ce pays dont la population totale est estimée à environ 3500000 individus. Le souhait des gouvernements libanais successifs de voir les réfugiés palestiniens quitter leur territoire - ce qu'ils nomment le 'refus de l'implantation' - est donc lié à des considérations de politiques internes, tout autant, voire plus, qu'à la recherche d'une solution durable et juste au conflit israélo-palestinien.

Les autorités libanaises ont mis en place un arsenal législatif qui vise à exclure les Palestiniens de toute forme d'intégration au Liban. La classe politique libanaise est aujourd'hui unanime pour refuser toute mesure qui pourrait favoriser l'intégration des Palestiniens, la presse libanaise se faisant souvent l'écho de cette question. La question palestinienne resurgit d'ailleurs de façon épisodique dans les médias lorsque, par exemple, le ministère de l'intérieur a demandé la révision des dossiers de naturalisation promulgués en 1994, les Palestiniens étant les premiers visés par cette requête ${ }^{11}$. Maintenus en marge de l'économie, du système de protection sociale - certes restreint - et de la vie politique libanaise depuis le départ de l'OLP, les réfugiés palestiniens se trouvent marginalisés au Liban plus que quand tout autre pays d'accueil de la diaspora. Un nombre croissant de réfugiés, s'ils ne demandent pas leur installation définitive au Liban, réclament le respect de leurs droits élémentaires, comme un accès sans discrimination au marché du travail, à l'éducation et au système de protection sociale. L'absence de perspectives d'intégration au Liban, comme l'arrêt du processus de paix, poussent un nombre croissant de réfugiés à chercher un autre pays d'accueil, plus propice à une installation sur le long terme ${ }^{12}$.

\footnotetext{
${ }^{9}$ UNRWA : Office de secours et de travaux des Nations Unies pour les réfugiés de Palestine dans le ProcheOrient, créé en 1949 par l'ONU pour venir en aide aux réfugiés palestiniens installés en Jordanie, Syrie, Liban, Cisjordanie et à Gaza.

${ }^{10}$ Marie-Louise Weighill ("Palestinians in Lebanon : the politics of assistance" in Journal of Refugee Studies, vol. 10, n³, Septembre 1997, pp. 294-313) analyse les effets du conflit libanais sur le recul de l'aide apportée à la population palestinienne.

${ }^{11}$ Le quotidien l'Orient - Le jour a titré "Près de 23000 réfugiés palestiniens vivent dans l'angoisse de perdre la nationalité libanaise " (19/05/2003).

${ }^{12}$ Cf. M. K. Doraï, "Palestinian Emigration from Lebanon to Northern Europe: Refugees, Networks, and

Transnational Practices" in Refuge (York University, Toronto), 21-2, Avril 2003, pp. 23-31.
} 
Le statut juridique des Palestiniens a d'importantes implications sur l'organisation socio-spatiale de cette communauté au Liban. Les réfugiés tendent à être confinés dans le secteur informel ou dans les activités les moins rémunératrices qui ne nécessitent pas l'obtention préalable d'une autorisation de travail ${ }^{13}$. De plus, le départ de l'OLP en 1982 a privé nombre de réfugiés des emplois induits par la forte présence des institutions politiques palestiniennes au Liban. Dans un contexte économique libanais en proie à de nombreuses difficultés depuis la fin de la Guerre civile, les Palestiniens se trouvent marginalisés sur le marché du travail au libanais, fortement concurrentiel avec l'arrivée d'une importante main d'œuvre étrangère. L'économie des camps est donc en récession.

\subsection{Une forte concentration de population dans les camps.}

La population palestinienne se trouve actuellement concentrée sur le littoral palestinien autour des principales villes libanaises littorales, Tyr, Saïda, Beyrouth et Tripoli, mais aussi dans une moindre mesure dans la plaine de la Beqaa autour des villes de Baalbek et de Zahlé (carte 2). Beyrouth et le Mont Liban forment toujours la plus grande concentration palestinienne avec 118879 réfugiés immatriculés à l'UNRWA. Tyr et Saïda sont ensuite les deux plus importantes zones de peuplement palestinien avec près de 90000 réfugiés inscrits pour chacune d'entre elles. La zone du sud Liban qui comprend ces deux villes concentre la moitié du total des Palestiniens au Liban.

Plus de la moitié des réfugiés inscrits vivent jusqu'à aujourd'hui dans des camps de réfugiés. Cette forte proportion de réfugiés qui vivent dans les camps est un trait caractéristique de la population palestinienne du Liban depuis son arrivée en 1948. Beyrouth forme la seule exception, puisque l'on y trouve seulement 2,9\% d'habitants dans les camps. Ce chiffre bas peut s'expliquer par l'origine urbaine des Palestiniens qui y résident. La population des camps est en effet essentiellement formée de paysans, les Palestiniens des villes ont par contre le plus souvent trouvé refuge en 1948 dans les villes libanaises, parce qu'à l'époque ils disposaient souvent de revenus suffisants pour y louer une habitation. Il faut ajouter à cela que les camps sont le plus souvent implantés en dehors des limites municipales des villes (seul le camp de Mar Elias se trouve aujourd'hui dans Beyrouth). Le fort pourcentage de résidents des camps à Tripoli, 85\%, peut s'expliquer lui par le fait que cette ville est une zone d'implantation très ancienne des réfugiés qui se dirigeaient alors vers la Syrie et qui ont été bloqués à la frontière. Ils se sont donc installés massivement dans les camps, faute d'argent mais aussi parce que stoppés dans leur parcours migratoire vers le nord de la Syrie, ils n'avaient pas d'autre alternative. Les régions de Tyr et de Saïda concentrent à elles seules près de la moitié de la population des camps du Liban, avec à elles deux 110000 réfugiés dans les camps. Pour la région de Tyr, la plus

\footnotetext{
${ }^{13}$ W. Said, "The obligations of host countries to refugees under international law : The case of Lebanon" in Naseer Aruri (ed.) Palestinian refugees. The right of return, London, Pluto Press, 2001, p. 123-151.
} 
Carte 2

La région de Tyr dans la distribution spatiale actuelle des Palestiniens au Liban.

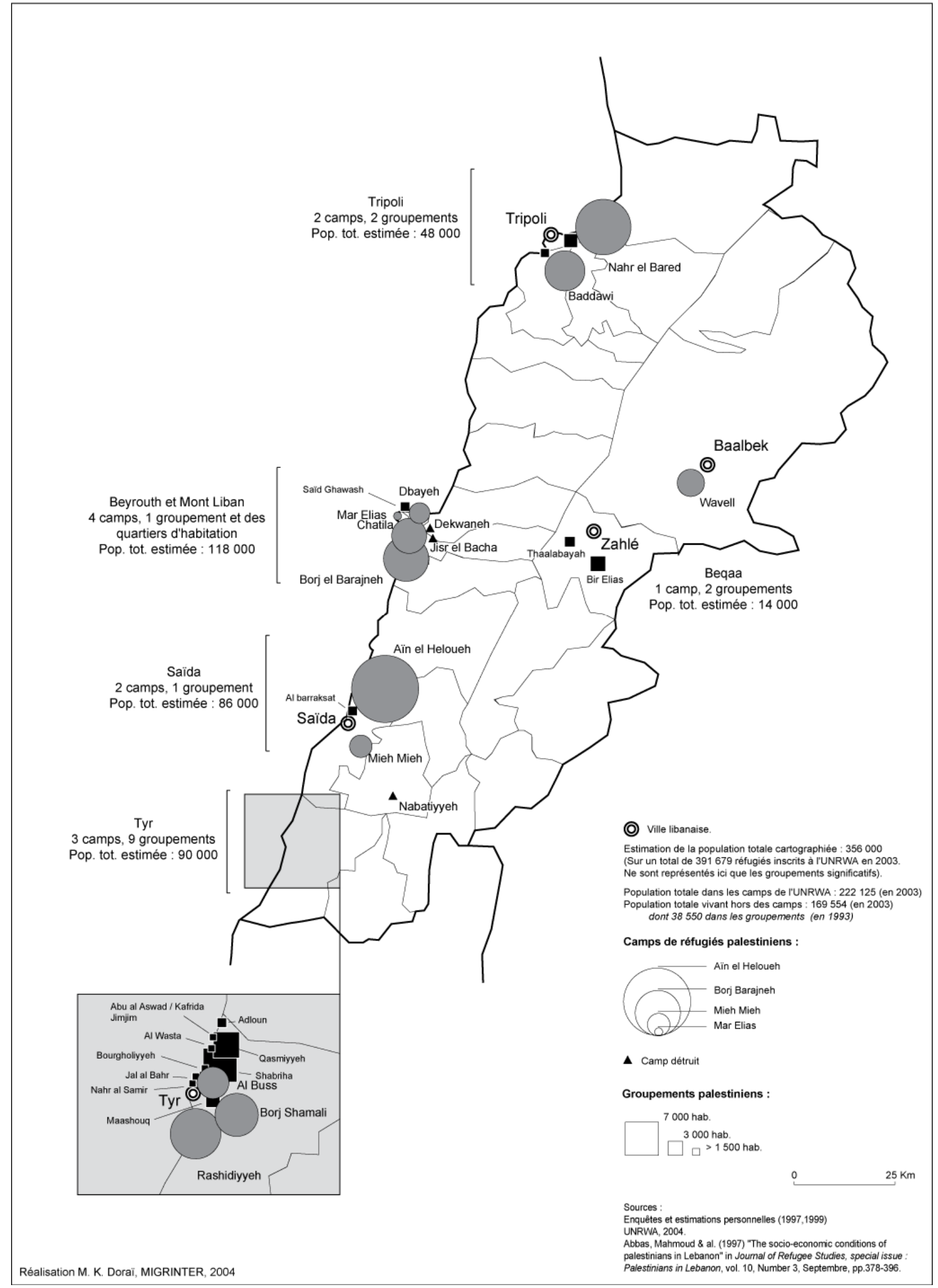


faible proportion de réfugiés dans les camps (54\%) qu'à Tripoli s'explique par la présence de nombreux groupements informels.

Tableau 1 : Les réfugiés palestiniens inscrits à l'UNRWA dans et hors les camps en juin 2003.

\begin{tabular}{lcccc}
\hline Région & $\begin{array}{c}\text { Population } \\
\text { résidente dans } \\
\text { les camps }\end{array}$ & $\begin{array}{c}\text { Population } \\
\text { résidente hors } \\
\text { des camps }\end{array}$ & $\begin{array}{c}\text { Population } \\
\text { totale }\end{array}$ & $\begin{array}{c}\text { \% de résidents } \\
\text { dans les camps }\end{array}$ \\
\hline Beyrouth & 1413 & 47004 & 48417 & 2,9 \\
Mont Liban & 45589 & 32811 & 78400 & 52,8 \\
Saïda & 56898 & 37942 & 94840 & 60 \\
Tyr & 53720 & 45684 & 99404 & 54 \\
Tripoli & 46658 & 8177 & 54835 & 85 \\
$\begin{array}{l}\text { Beqaa } \\
\text { Autres réfugiés }\end{array}$ & 7492 & 8291 & 15783 & 41,5 \\
immatriculés. & - & - & 10355 & - \\
& 211770 & 179909 & 391679 & 55,5 \\
\hline Total & & & \multicolumn{2}{c}{ Source : UNRWA (2004). }
\end{tabular}

Sur seize camps de réfugiés au Liban, trois ont été détruits pendant la guerre civile, Nabatiyeh au sud Liban, Tel Zaatar et Jisr al Basha dans la région de Beyrouth. Treize camps existent donc encore aujourd'hui. La majeure partie d'entre eux ont été construits dans les premières années d'arrivée des Palestiniens entre 1948 et 1955, à l'exception de Rashidiyyeh et Al Buss, qui étaient des espaces prévus pour l'accueil des Arméniens ${ }^{14}$ et construits en 1936, puis utilisés pour accueillir les Palestiniens en 1948, alors que presque toutes les familles arméniennes les avaient quittés pour s'installer en ville. La superficie des camps est très variable, elle est fonction de deux facteurs principaux. D'abord, le lieu d'implantation. Les camps ruraux comme Rashidiyyeh ou Borj Shamali sont de taille conséquente (respectivement 367200 m² et $134600 \mathrm{~m}^{2}$ ) parce que l'espace disponible est plus important. Les camps urbains sont eux moins étendus, comme Shatila (39 567 m²). Généralement le bâti y est beaucoup plus dense. De l'autre, le nombre de réfugiés à la création. Aïn el Heloueh (301 $039 \mathrm{~m}^{2}$ ) ou Nahr al Bared (198 129 m²) accueillaient dès leur création d'importantes communautés palestiniennes, fortes de plusieurs milliers de réfugiés.

\footnotetext{
${ }^{14}$ De 1915 à 1918, la population arménienne fait face à des massacres et des déportations de la part des Turcs. De nombreux Arméniens se dirigent vers la Syrie et le Liban pour trouver refuge. Ils s'installent au Liban et se voient octroyer la nationalité de leur pays d'accueil. Cette communauté d'un peu moins de 200000 personnes représente aujourd'hui près de $5 \%$ de la population libanaise.
} 
Tableau 2: Les camps de l'UNRWA au Liban : établissement, superficie et population en juin 2003.

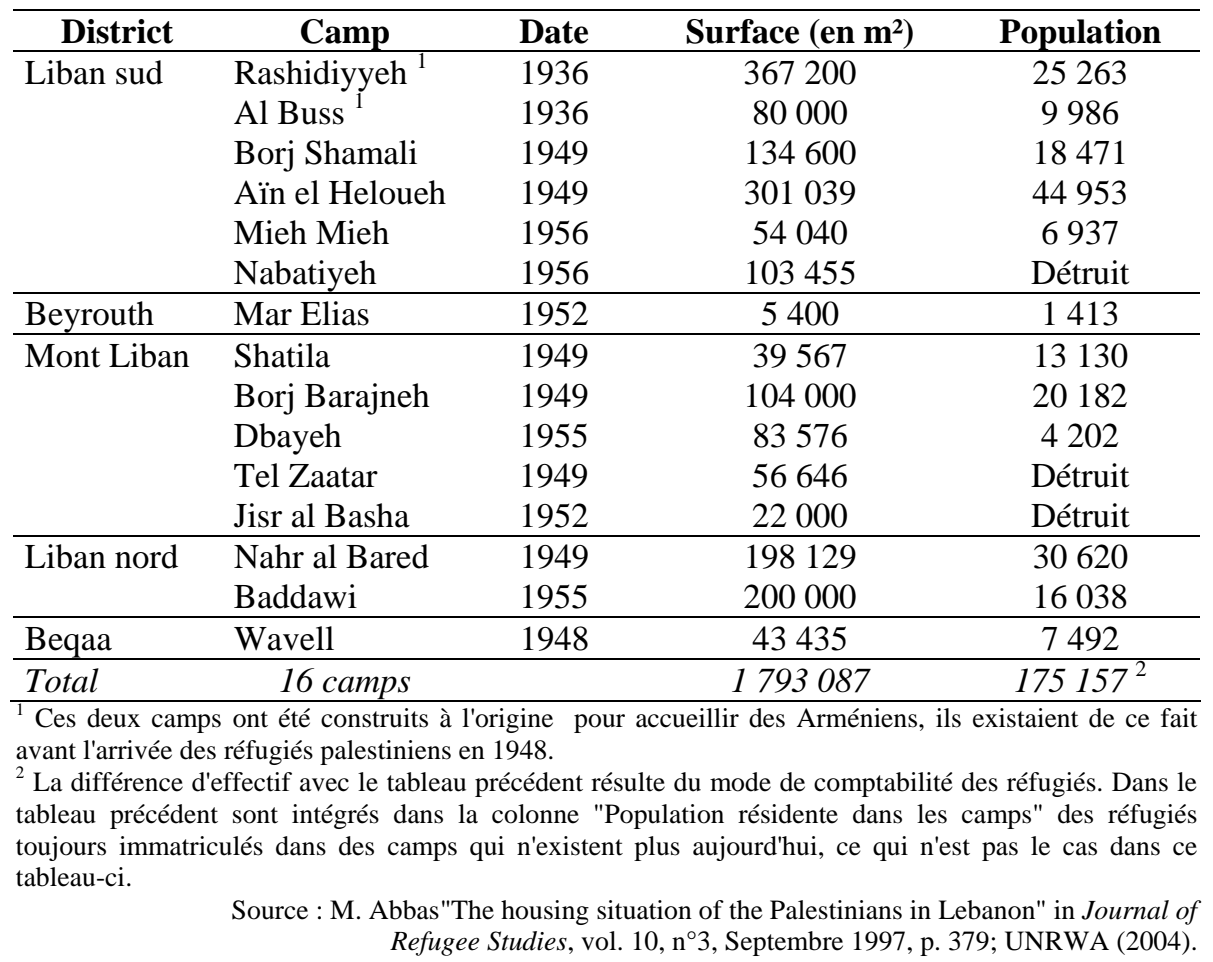

Le camps le plus peuplé est Aïn el Heloueh à l'entrée de Saïda avec près de 40000 habitants ${ }^{15}$, vient ensuite Nahr el Bared avec plus de 25000 habitants près de Tripoli, au nord du Liban, et Rashidiyyeh au sud de la ville de Tyr, avec plus de 22000 habitants. Les camps les plus importants se situent dans les principales zones de résidence des Palestiniens. Les principaux camps de réfugiés se situent à proximité des centres urbains libanais, surtout lorsque ces villes comportent une importante communauté sunnite, comme c'est le cas pour Saïda, Tripoli et dans une certaine mesure Beyrouth. Ces villes littorales sont aussi des espaces économiques dynamiques, les réfugiés sont donc susceptibles d'y trouver plus facilement un emploi. Le développement du camp de Rashidiyyeh, le camp rural le plus peuplé, est lié lui à sa situation au cœur de la zone de transit des réfugiés palestiniens qu'est le sud Liban. C'est aussi un bassin d'emplois agricoles, dans les vergers et les cultures maraîchères qui se développent le long de la plaine littorale, autour du village libanais de Ras al Aïn par exemple.

\section{Les camps de réfugiés à Tyr : aux marges de la ville ?}

La structure actuelle des camps et des groupements palestiniens ne s'est pas fortement modifiée depuis le milieu des années soixante (carte 3). Ni la guerre civile libanaise (1975-1991), ni les deux invasions

\footnotetext{
${ }^{15}$ Ces chiffres fournis par l'UNRWA sous-estiment de façon très importante la population résidente dans ce camp, puisque seuls les personnes officiellement enregistrées par l'UNRWA dans ce camp sont comptabilisées. Dans la réalité Aïn el Heloueh compterait plus de 80000 habitants.
} 
Carte 3

Les camps et groupements de la région de Tyr en 1997.

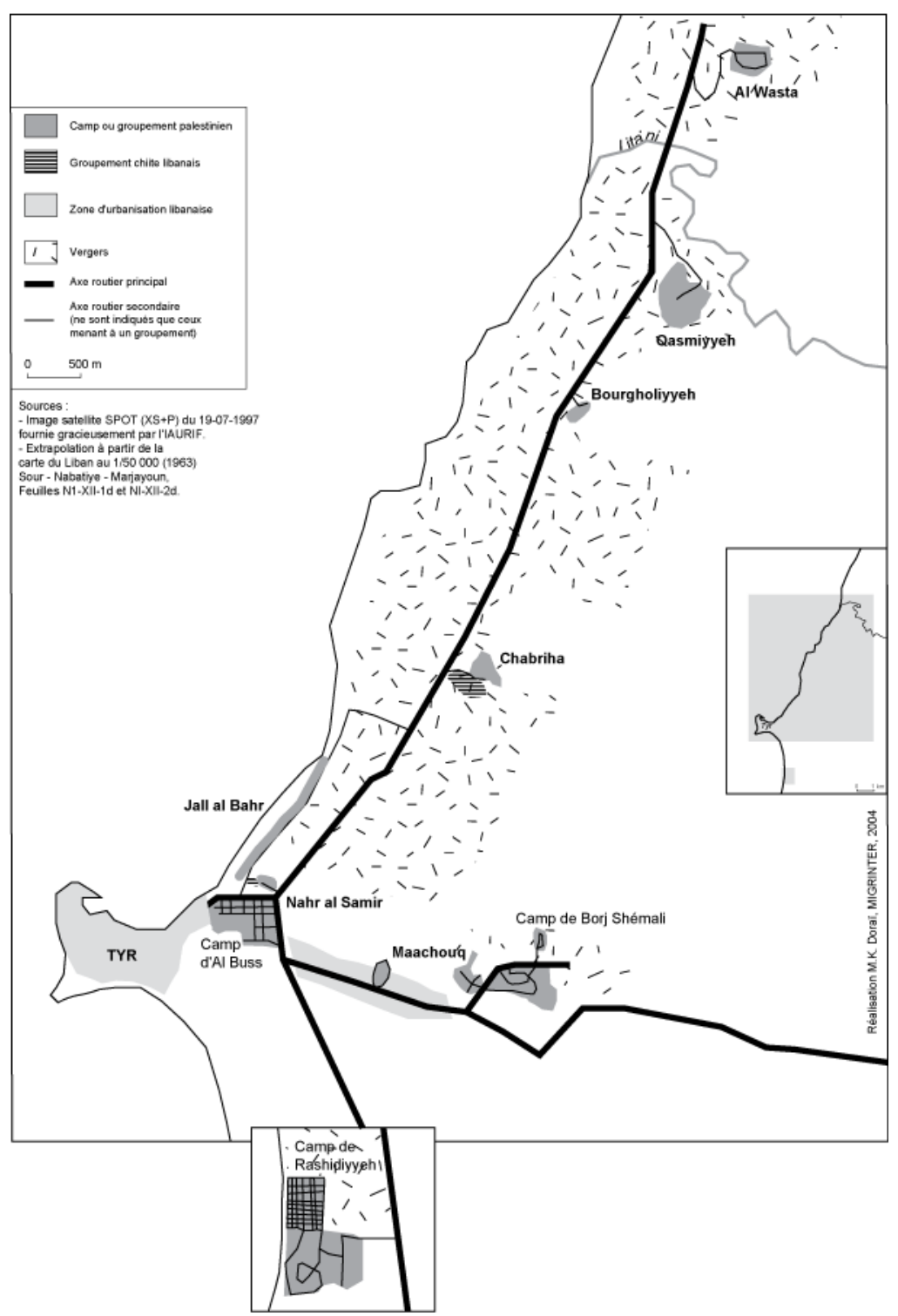


israéliennes (1978 et 1982), ni la Guerre des camps (1985-1987) n'ont modifié de façon importante les espaces occupés par les réfugiés palestiniens. La région de Tyr n'a pas connu de destructions de camps comme à Beyrouth ou à Nabatiyeh. Si la population palestinienne a été animée d'une forte mobilité durant ces épisodes de guerre, dans la plupart des cas elle est toujours venue se réinstaller là où elle vivait précédemment.

\subsection{Une urbanisation récente.}

Dans les années quarante, avant l'arrivée des réfugiés palestiniens, la ville de Tyr est confinée au nord ouest de la presqu'île. Elle est composée de la vieille ville et d'une extension plus récente qui l'entoure. Elle se développe essentiellement autour de son port. Les camps d'Al Buss et de Rashidiyyeh se trouvent dans la campagne. Les Arméniens qui y habitent quittent petit à petit ces espaces, pour se diriger vers Beyrouth. La ville de Tyr est entourée d'espaces agricoles parsemés de petits villages. Dans les années cinquante et soixante, la ville de Tyr s'étend vers l'est jusqu'à occuper l'ensemble de la presqu'île, à l'exception de sa partie sud-ouest occupée par une vaste zone archéologique. Le camp palestinien d'Al Buss qui se développe par le regroupement de réfugiés palestiniens et par accroissement naturel se retrouve à l'entrée de l'agglomération de Tyr. On assiste en fait au mitage de l'espace compris entre la ville et le camp.

L'ensemble des espaces bâtis jusque dans les années soixante est toujours présent autour de la ville de Tyr. Une promenade a été construite en l'an 2000 le long du rivage nord qui borde Tyr, et relie ainsi la partie ancienne de la ville au rond point d'Al Buss. Le camp se trouve donc intégré de facto dans l'aménagement du littoral. Le camp de Borj Shémali, ainsi que le groupement de Maachouq, isolés à leur création, se trouvent aujourd'hui enserrés par une urbanisation en ruban qui se développe le long de l'axe routier qui part de la ville de Tyr et se dirige vers l'intérieur des terres. Ces quatre espaces, à l'origine ruraux, se trouvent donc intégrés de fait à des degrés divers à l'entité urbaine de Tyr. Le camp de Rashidiyyeh, situé lui au sud de l'agglomération, n’a pas été rattrapé par les constructions et conserve pleinement son caractère rural. Il est entouré de zones agricoles. Les groupements ruraux qui se situent le long de l’axe routier qui relie Tyr à Saïda, sont toujours situés en milieu rural et environnés de vergers.

Aujourd'hui, le camp d'Al Buss se trouve enserré dans l'espace urbain de Tyr, puisque des zones d'habitations libanaises se sont développées au nord du camp, le long de l'axe routier qui mène à Saïda. De la même façon, une urbanisation en ruban s'est développée vers l'est le long de l'axe qui relie Tyr à Borj Shémali, intégrant le groupement de Maachouq et le camp de Borj Shémali dans l'espace de la ville de Tyr. Aujourd'hui donc deux camps, celui d'Al Buss et celui de Borj Shémali, et trois groupements, Nahr al Samir, Maachouq et Jall al Bahr, font partie de l'agglomération de Tyr. 


\subsection{Le faible dynamisme spatial des camps et des groupements.}

A l'échelle de la région de Tyr, l'espace occupé par les zones d'implantations palestiniennes ne s'est ni accru, ni densifié au cours de ces dernières années. Ceci est essentiellement dû à la stagnation relative du nombre de Palestiniens résidant dans la région de Tyr. La mobilité interne au Liban, vers Saïda et Beyrouth, ainsi que la mobilité internationale, essentiellement vers l'Europe du nord, limite la croissance de la population palestinienne, voire engendre son déclin progressif. Aujourd'hui, autour de Tyr, on compte toujours aujourd'hui autour de Tyr trois camps de réfugiés et neuf groupements informels.

\subsubsection{Des espaces en voie d'intégration au tissu urbain de Tyr.}

Rares sont les zones d'habitations palestiniennes qui continuent de se développer, même lentement. Seuls se trouvent dans cette situation les deux espaces à dominante urbaine qui jouxtent l'agglomération de Tyr, le groupement de Nahr al Samir et le camp d'Al Buss. Ces deux espaces sont aujourd'hui intégrés à l'entité urbaine de Tyr. Ils ne sont plus séparés de la ville, mais le rond point d'Al Buss sur lequel ils se trouvent constitue aujourd'hui l'entrée nord de la ville par laquelle arrive la route qui vient de Saïda. L'habitat a donc tendance à être amélioré voire à se développer, par la construction de nouveaux étages par exemple. Deux facteurs permettent d'expliquer ce dynamisme relatif : (1) le camp et le groupement, même s'ils constituent des espaces fermés et délimités spatialement, s'intègrent dans une certaine mesure au tissu urbain de Tyr. Il est par exemple de plus en plus difficile de repérer la limite sud ouest du camp. On assiste à un mitage de l'espace compris entre ce dernier et la ville de Tyr, par des habitations individuelles, souvent construites par des Chiites libanais exilés de la zone sud pendant l'occupation israélienne. Les nombreux commerces qui se sont implantés le long des axes routiers au nord du camp comme à l'est, possédés tant par des Palestiniens que par des Libanais, intègrent la frange extérieure du camp dans le paysage urbain. (2) Le dynamisme économique, surtout commercial, de ces espaces, par les revenus qu'il procure, permet aux Palestiniens d'améliorer leur habitat, qui tend de plus en plus à ressembler à celui des quartiers pauvres ou des classes moyennes libanaises peu favorisées. Les quartiers d'habitat informel ont tendance à évoluer en s'intégrant à l'activité économique et au paysage urbain de la ville de Tyr. C'est la situation du camp d'Al Buss comme du groupement de Nahr de Samir, à l'entrée de la ville, à un carrefour de communication qui favorise cette évolution.

\subsubsection{Des espaces marginalisés.}


La majeure partie des camps et des groupements se développe au ralenti aujourd'hui. C'est ce que l'on constate pour les deux autres camps de réfugiés, Rashidiyyeh et Borj Shémali, ainsi que le reste des groupements. La plupart de ces espaces ont connu des destructions pendant la Guerre des camps, toujours visibles aujourd'hui. Dans la nouvelle partie du camp de Rashidiyyeh on voit jusqu'aujourd'hui des façades de maisons criblées de balles, ainsi que des murs détruits par des tirs de roquettes. Certaines habitations, trop détruites et dont les habitants ont décidé de partir définitivement vivre ailleurs au Liban mais surtout à l'étranger, demeurent inhabitées et tombent en ruines. Le groupement de Jall al Bahr a été détruit en partie à cette même époque. Une vingtaine d'habitations ont été entièrement détruites au nord du groupement, entraînant dans son sillage le départ de plusieurs dizaines de réfugiés vers Saïda, et bon nombre d'entre eux ont ensuite émigré vers le Danemark. D'autres habitations portent encore les marques de la guerre, parce que leurs habitants ne possèdent pas l'argent nécessaire à leur réparation ou bien parce que les Autorités libanaises refusent leur réhabilitation.

De façon plus générale, même si tous les espaces ont subi des destructions pendant la Guerre des camps, aujourd'hui il ne reste plus que quelques traces matérielles ponctuelles. Les deux camps de réfugiés cités connaissent à l'heure actuelle une phase de stagnation et ce en raison de deux facteurs principaux : (1) l'espace alloué aux camps est délimité géographiquement, et les Autorités libanaises veillent scrupuleusement à ce que les camps ne s'étendent pas. Le camp de Borj Shémali ne dispose pas de cimetière, les défunts sont donc enterrés à Maachouq. Les responsables du camp ont cependant émis le projet de bâtir un cimetière au nord du camp, sur des terres qui leur reviennent selon eux. Les Libanais, estiment pour leur part que cet espace ne fait pas partie du camp et refusent donc l'installation du cimetière à cet endroit. (2) Rashidiyyeh et Borj Shémali relativement isolés spatialement ne se trouvent pas dans des zones économiques dynamiques qui leur permettraient de se développer.

Pour les groupements de la région de Tyr la situation est assez similaire à celle des camps. Leur développement est entravé pour trois principaux motifs. Etablis de façon informelle et le plus souvent sans titre de propriété, voire sans l'accord des propriétaires lorsqu'il y en a, leur espace de développement est forcément réduit aux terres qu'ils occupent déjà. L'ensemble des groupements qui se situent au nord de Chabriha sont isolés au milieu de zones de vergers, leur dynamisme économique est faible parce que la majeure partie des habitants sont des ouvriers agricoles journaliers, le commerce et l'artisanat y sont presque totalement absents. Par ailleurs, leur implantation sur des pentes rocheuses rend de toutes les façons leur extension spatiale difficile.

3.2.3. Al Buss, un camp de plus en plus intégré au tissu urbain de Tyr ? 
Les limites nord et est du camp d'Al Buss sont plus densément bâties que les autres quartiers (carte 4). De fait les constructions sont mitoyennes les unes des autres. La route qui longe le camp au nord mène vers le centre ville de Tyr, celle qui le borde à l'est se dirige vers le sud Liban ou bien l'intérieur des terres. C'est une zone de trafic intense vers laquelle convergent les flux de voyageurs. Le rond point d'Al Buss (Duwwar Al Buss) qui se situe à l'intersection de ces deux axes, fait office de gare routière, où stationnent les bus et les taxis collectifs qui se rendent à Saïda ou Beyrouth, et depuis mai 2000 et le retrait israélien, vers le sud Liban. Cette zone d'habitat est devenue zone commerciale, où l'on trouve tous types d'épiceries et d'artisans.

La zone d'habitation peut être subdivisée en deux espaces distincts. En premier lieu la partie qui s'est développée autour de l'ancien camp arménien. Il s'agit d'un espace assez densément construit. Plus on s'éloigne de la bordure du camp, moins l'espace bâti est dense. Le maximum de densité se trouve au nord-est du camp. Les habitations sont le plus souvent contiguës et à deux étages. Plus à l'intérieur, les habitations sont plus espacées, et ne disposent que plus rarement d'un étage supplémentaire. On peut noter la présence de jardins autour des habitations. Dans cette partie du camp, les rues sont assez larges et se coupent à angle droit. Elles dessinent donc des îlots rectangulaires plus ou moins vastes.

En deuxième lieu, la partie ouest du camp s'est développée de façon informelle. Plus on se dirige vers le sud-ouest du camp, moins l'habitat est dense. Les ruelles qui séparent les habitations sont étroites et tortueuses. Cette extension est surtout habitée par des Palestiniens originaires de Damoun, qui se sont pour partie spécialisés dans la culture des vergers. Un certain nombre d'entre eux ont dégagé d'importants profits de cette activité et disposent donc de maisons à un ou deux étages avec une cour intérieure. On rencontre aussi de l'habitat plus modeste, de type individuel de plain-pied entouré d'un jardin potager. Entre ces deux parties du camp se situent les écoles de l'UNRWA, qui occupent une superficie très importante, étant donné l'investissement fait par l’UNRWA et le nombre d'enfants réfugiés à scolariser.

Le camp d'Al Buss présente une particularité par rapport aux deux autres camps de Tyr, ainsi qu'aux autres camps au Liban, il comporte en son sein deux espaces non-palestiniens : un hôpital public libanais ainsi qu'une église maronite. Ces deux espaces sont en fait des héritages arméniens et chrétiens libanais, le premier hôpital de Tyr aurait été construit par les Arméniens d'Al Buss, et l'église ayant été construite sur une terre qui appartient à la communauté religieuse maronite. Cet édifice religieux a une histoire particulière, racontée par l'ancien maire arménien du camp. L'église actuelle est en fait relativement récente. Le projet de construire une église était lui ancien, mais faute d'argent, elle n'avait pas vu le jour. Dans les années quatre-vingt, les habitants du camp, dont la quasi-totalité sont musulmans, se sont cotisés pour construire cette église fréquentée aujourd'hui par des chrétiens libanais tous les samedis. Pendant la Guerre des camps, alors qu'Al Buss était soumis à un blocage 
Carte 4

Le camp d'Al Buss : un espace sous contrôle

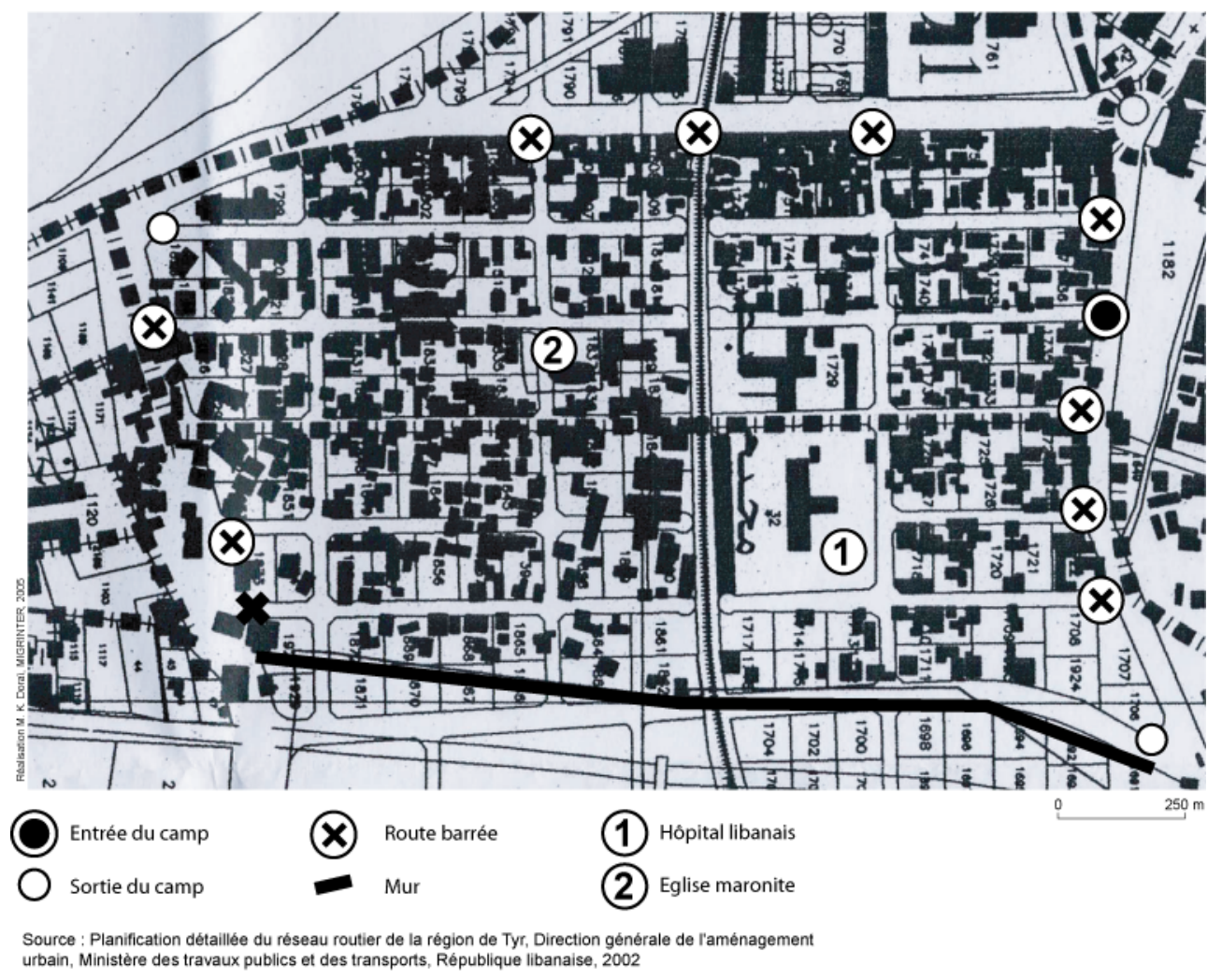

presque total par les miliciens chiites libanais d'Amal, c'était les fedayin palestiniens qui assuraient la sécurité des chrétiens pour chaque office religieux. Ce camp incarne spatialement l'imbrication des communautés, palestiniennes et libanaises d'un côté, musulmanes et chrétiennes de l'autre.

Aujourd'hui ce camp est soumis à un contrôle strict de la part de l'armée libanaise. L'ensemble des ruelles qui débouchent sur les routes qui contournent le camp sont obstruées, et ne permettent que de passer à pied. Les voitures ne disposent que d'une seule entrée, et d'une seule sortie, sur lesquelles l'armée veille jour et nuit.

Le long des deux axes qui entourent le camp, les activités sont pratiquées tant par des Palestiniens que par des Libanais. Il s'agit donc d'une zone d'activité mixte. Celle-ci s'étend d'ailleurs tout autour du rond point par des restaurants, des cafés, et des commerces divers. A l'intérieur du camp, seuls les Palestiniens exercent une activité. Le camp comprend toutes les activités classiques et nécessaires pour vivre : restaurant, photographe, épicier, coiffeur, vendeur de fruits et légumes, électricien, menuisier, etc... Le camp d'Al Buss est le seul camp intégré dans l'espace urbain de Tyr. Son développement économique est lié à cette situation particulière qui dynamise les espaces de contact - les frontières externes - avec l'environnement libanais. L'activité économique d'Al Buss se prolonge dans le 
groupement voisin de Nahr al Samir. Il faut rappeler que la majeure partie des réfugiés qui vivent ici est originaire de la ville de Haïfa. Ils sont donc issus de familles commerçantes et non pas paysannes comme la majeure partie des réfugiés de la région de Tyr. Les activités se développent autour du rond point $\mathrm{Al}$ Buss qui marque l'entrée de la ville.

\section{Conclusion.}

L'inscription de la diaspora dans ses espaces d'accueil avec la mise en place des camps de réfugiés a permis la constitution dans l'exil de regroupements familiaux et villageois, à l'image de ceux préexistants dans la société palestinienne qui ont précédé l'exode. La Palestine joue toujours un rôle symbolique structurant dans l'organisation en exil de l'espace palestinien. L'évolution socio-spatiale des camps est impulsée par les réfugiés eux-mêmes, supportée par leurs institutions diasporiques et aidée par l'UNRWA, ceci dans les limites imposées par l'Etat libanais et les acteurs hostiles à la présence palestinienne. Les camps, lieux de résidence des réfugiés, occupent une place particulière dans la géographie palestinienne de l'exil, parce qu'ils sont des lieux de mémoire et parce qu'ils inscrivent dans l'espace de façon pérenne l'exode de 1948 ainsi que la permanence de la question des réfugiés. Ils ont permis le maintien et l'adaptation des systèmes de solidarité traditionnels, qui sont l'une des principales clés de compréhension de l'organisation de l'espace palestinien en diaspora.

Le développement spatial des camps, ainsi que le rôle qu'ils jouent dans la société palestinienne est lié au développement des institutions diasporiques palestiniennes au Liban. Après les Accord du Caire signés en 1969 entre l'OLP et les autorités libanaises les camps deviennent des espaces sous juridiction palestinienne. Dans l'exil, outre la fonction résidentielle, les camps et les groupements remplissent trois rôles au sein de la société palestinienne. Ils sont les lieux d'expression des formes de solidarités villageoises et familiales. Ils assurent aussi la recomposition de l'identité palestinienne malgré la dispersion, parce qu'ils inscrivent dans la géographie la nakba. De plus, ce sont des espaces d'expression politique, surtout après la signature des Accords du Caire, puisque dans ces lieux les réfugiés palestiniens bénéficient d'une certaine liberté politique. Ces espaces forment donc le tissu de base de la société palestinienne dans l'exil. Cependant, au fur et à mesure de la perte d'influence de la centrale palestinienne au Liban, les camps deviennent des espaces de repli identitaire, social et économique. Avec l'augmentation de la pression démographique, et le manque de moyens financiers, les infrastructures sont sous-adaptées et les habitations sont en voie de délabrement. La crise économique que traversent les réfugiés palestiniens, qui découle directement des contraintes juridiques qui leurs sont imposées par les autorités libanaises, est l'un des facteurs qui permet de comprendre la situation dans laquelle se trouvent aujourd'hui les camps et les groupements. 